\title{
Restricted Palivizumab Recommendations and the Impact on RSV Hospitalizations among Infants Born at $>29$ Weeks of Gestational Age: An Italian Multicenter Study
}

\author{
Elena Priante, $\mathrm{MD}^{1, *} \quad$ Elena Tavella, $\mathrm{MD}^{2, *} \quad$ Elisa Girardi, $\mathrm{MD}^{3} \quad$ Maria Angela Militello, $\mathrm{MD}^{2}$ \\ Veronica Mardegan, MD ${ }^{1}$ Milena M. Maule, MD, PhD ${ }^{4}$ Alberto Dall'Agnola, MD \\ Eugenio Baraldi, MD, $\mathrm{PhD}^{1,6}$ Paolo Manzoni, MD²,6
}

\footnotetext{
${ }^{1}$ Department of Woman's and Child's Health, Unit of Neonatal Intensive Care, University of Padua, Padua, Italy

2 Division of Pediatrics and Neonatology, Department of Maternal and Infant Medicine, Nuovo Ospedale degli Infermi, ASL Biella, Italy

3 Paediatrics and Neonatology, Hospital Villafranca di Verona, Italy

${ }^{4}$ Department of Medical Sciences, Cancer Epidemiology Unit,

University of Turin, Turin, Italy

${ }^{5}$ Neonatology and Pediatric Infectious Disease, P. Pederzoli Hospital, Peschiera del Garda, Regione Veneto, Italy

6 Respiratory Syncytial Virus Network (ReSViNET) Foundation, Zeist, The Netherlands
}

Am J Perinatol 2019;36(suppl S2):S77-S82.
Address for correspondence Paolo Manzoni, MD, Division of Pediatrics and Neonatology, Department of Maternal and Infant Medicine, Nuovo Ospedale degli Infermi, Via dei Ponderanesi 2, Ponderano, Biella, Italy (e-mail: paolomanzoni@hotmail.com).

\section{Abstract \\ Keywords \\ - respiratory syncytial virus \\ - palivizumab \\ - chronic lung disease}

Objective Premature infants have the highest risk of being hospitalized with respiratory syncytial virus (RSV) infections. Palivizumab is the only licensed agent for RSVhospitalization (RSVH) prophylaxis in infants born at $<35$ weeks of gestational age (WGA). In 2016, the Italian Drug Agency (Agenzia Italiana del Farmaco [AIFA]) has restricted the eligibility for reimbursement to infants at high risk of RSVH, ruling out palivizumab administration for infants born at $>29$ wGA. The aim of the present study was to compare the incidence of RSVH in two consecutive epidemic seasons (2015-2016 vs. 2016-2017), that is, before and after the new AIFA recommendations on palivizumab eligibility.

Study Design This was a noninterventional retrospective cohort study conducted at three neonatal intensive care units (NICUs) in northern Italy. Infants born at 29 and 35 wGA between March 15, 2015 and March 14, 2017 were enrolled for this study. Electronic medical charts were reviewed and parents were interviewed by telephone. Data were collected on neonatal course during NICU stay, palivizumab administration, and hospitalizations related to respiratory infections during the 1st year of life, comparing the infants born in season 1 with season 2 .

Results Of 632 eligible infants, data were available for 536 ( 262 in season 1 and 274 in season 2). Overall, RSVH occurred 1.9 and $5.1 \%$ in infants in seasons 1 and 2 , respectively (odds ratio $[\mathrm{OR}]=2.77 ; 95 \%$ confidence interval $[\mathrm{Cl}]$ : $0.98-7.8$, $p=0.045$ ). When the analysis was limited to patients not exposed to palivizumab, RSVHs were recorded for 1.8 and $5.9 \%$ infants in seasons 1 and 2, respectively

Elena Priante and Elena Tavella contributed equally as first authors of this article.

Copyright (c) 2019 by Thieme Medical Publishers, Inc., 333 Seventh Avenue, New York, NY 10001, USA. Tel: +1(212) 584-4662.
DOI https://doi.org/ 10.1055/s-0039-1691771. ISSN $0735-1631$. 
( $\mathrm{OR}=3.42 ; 95 \% \mathrm{Cl}: 0.96-12.20, p=0.045)$. It is noteworthy that the incidence of hospital admissions for respiratory viruses other than RSV did not differ between the two seasons.

Conclusion Restricting eligibility for palivizumab reimbursement led to a significant increase in RSVH but had no impact on hospitalizations for other respiratory viruses. Future decisions on palivizumab prescription and coverage rules should be driven by a careful assessment of the cost-benefit ratio.

Respiratory syncytial virus (RSV) is the most common cause of lower respiratory illness among infants, resulting in annual epidemics worldwide. RSV infection is a significant health care issue for infants in the early months of life, and especially in those born prematurely. Hospitalizations and outpatient visits for community-acquired RSV infections are associated with significant health care costs and resource usage. The management of RSV-related illness has remained largely unchanged in the last decades, and is mainly supportive. The monoclonal antibody palivizumab is the only agent marketed and approved for RSV prophylaxis, but its use is restricted to high-risk children (preterm-born infants, children with chronic lung disease [CLD] of prematurity, children with hemodynamically significant congenital heart disease [HSCHD], and those with neuromuscular disease). ${ }^{1,2} \mathrm{~A}$ Cochrane's review published in 2013 concluded that palivizumab is effective in reducing the incidence of RSV-related hospitalizations (RSVH) for severe lower respiratory tract infections involving children with chronic lung disease or congenital heart disease, or infants born preterm. It is worth noting that the Cochrane's review considered all infants born preterm as a single group, irrespective of their gestational age at birth. ${ }^{3}$ Guidance for palivizumab prophylaxis from the American Academy of Pediatrics, updated in 2014, did not include preterm infants born at $>29$ weeks of gestation (wGA) in the recommendations to receive that treatment, unless there were comorbidities involved. ${ }^{4}$

In Italy, palivizumab was administered to all premature infants born at $<32 \mathrm{wGA}$ and also to those born at 33 to 35 wGA if they had certain additional risk factors up until 2016. Then the Italian Drug Agency (Agenzia Italiana del Farmaco [AIFA]) downgraded palivizumab reimbursement to include only premature infants born at $<29$ wGA. The concomitant update on the scientific recommendations issued by the Italian Society of Neonatology took a different approach, continuing to recommend palivizumab administration for infants born between 29 and 32 wGA. ${ }^{5}$ While it is hard to establish a definite gestational age "threshold" distinguishing between high-risk and low-risk infants, current evidence in the literature indicates that preterm infants born at 29 to $35 \mathrm{wGA}$ are all at risk of severe RSV-related disease than term-born infants, particularly in the early months of life. ${ }^{6}$ Lanari et al demonstrated that the risk estimates for hospital admissions due to bronchiolitis doubled for infants born at 33 to 34 wGA compared with those born at term. ${ }^{7}$ A recently-published observational cohort study, the SENTINEL study, investigated the burden of severe RSV-related disease in infants born at 29 to $35 \mathrm{wGA}$ who were not given palivizumab; it showed that earlier GA and younger chronological age were associated with the highest risk of severe RSV-related disease in terms of hospitalizations, admission to intensive care units, and need for mechanical ventilation. ${ }^{8}$ There is, therefore, a discrepancy between the scientific recommendations and the rules governing eligibility for palivizumab reimbursement. The scientific societies have encouraged the production of more data and further studies to clarify this conflicting issue.

With this perspective, we designed a study to compare the incidence of RSVH among infants born at 29 to 35 wGA in the season before (2015-2016) or after (2016-2017) the introduction in 2016 of the AIFA's more restricted recommendations for palivizumab.

\section{Materials and Methods}

This retrospective observational cohort study was conducted at three neonatal intensive care units (NICUs) in northern Italy. All Infants born at 29 to 35 wGA during two consecutive epidemic seasons were identified from birth registries and health service administrative data.

Season 1 included infants born at 29 to 35 wGA between March 15, 2015 and March 14, 2016. These infants would have received palivizumab based on the following criteria: (1) prematurity $<32^{6 / 7}$ wGA and $<12$ months old; (2) birth at 33 to $35 \mathrm{wGA}$ and additional risk factors (exposure to smoke, treatment with surfactant, living with siblings $<10$ years old, attendance at daycare), and $<12$ months old; (3) CLD and $<12$ months old; (4) CLD and $<24$ months old when medical therapy was required at the start of the season; (5) HSCHD and $<12$ months old; and (6) neuromuscular diseases, congenital abnormalities of the airways, or immune deficiency, and $<12$ months old.

Season 2 concerned infants born at 29 to 35 wGA between March 15, 2016 and March 14, 2017. This group would have received palivizumab in the case of: (1) prematurity $<29^{6 / 7}$ wGA and $<12$ months old; (2) CLD and $<12$ months old; (3) CLD and $<24$ months old when medical therapy was required at the start of the season; (4) CHD and $<12$ months old; and (5) neuromuscular diseases, or congenital abnormalities of the airways, or immune deficiency, and $<12$ months old.

The following information on the patient's neonatal characteristics and clinical course while at the NICU was obtained from electronic medical charts: GA and birth weight, need for 
mechanical ventilation, diagnosis of CLD or HSCHD, indications for palivizumab prophylaxis, number of doses, mode of administration, and compliance. Infants with major congenital anomalies other than HSCHD were excluded from the study.

Palivizumab (Synagis, AbbVie Srl, Aprilia, Italy) $15 \mathrm{mg} / \mathrm{kg}$ was administered in five monthly courses over the epidemic seasons (from late November to late March) according to existing protocols and guidelines. ${ }^{5}$ The same timing and dosage were used for all infants, regardless of their GA and/ or underlying conditions. None of the infants were treated out of season.

Cases of bronchopulmonary dysplasia (BPD) were identified from the medical records in compliance with the criteria stipulated by the consensus definitions of BPD. ${ }^{9}$ The HSCHD was defined as uncorrected, or palliated cyanotic or acyanotic CHD with pulmonary hypertension (systolic pulmonary arterial pressure $>40 \mathrm{~mm} \mathrm{Hg}$ ), or need for medication to manage congestive heart failure, and the diagnosis was confirmed by a pediatric cardiologist. ${ }^{10}$

Parents of eligible infants were interviewed by telephone through a questionnaire to collect additional information about the number and age of siblings at home, daycare attendance, breastfeeding, exposure to smoke, and hospitalizations for respiratory infections during the 1 st year of life. A risk factor score was calculated, scoring one point for each of the following: (1) more than two siblings at home; (2) daycare attendance; (3) no breastfeeding, or breastfeeding for less than 2 months; (4) parental smoking; and (5) discharge from the NICU in the epidemic season. When hospital admissions for respiratory infections were reported, parents were invited to provide all related medical records. A thorough review of this clinical documentation was performed to check whether these hospitalizations were related to RSV infection or not (as primary and secondary outcomes, respectively).

RSVHs were defined as symptomatic admissions to hospital for deep or wet chest cough, wheezing, hoarseness, stridor, or shortness of breath. RSV positivity was confirmed by enzyme or immunofluorescent assay, polymerase chain reaction, or a positive viral culture for RSV from nasopharyngeal secretions. ${ }^{11}$

For the purpose of this study, only the first episode of RSVH in each infant was considered. The infants' age at the time of their RSVH, and the need for mechanical ventilation or admission to the NICU were also recorded.

Informed consent for the collection of the infants' medical data was obtained from parents shortly after birth and confirmed at the time of the telephone interview.

\section{Statistical Analysis}

The demographic and clinical data examined are reported as counts (percentages) for categorical variables and mean \pm standard deviation (SD) for normally-distributed continuous variables. Proportions and continuous variables were compared using Fisher's exact two-tailed test and the $t$-test, respectively. Specifically, the association between RSVH and season (classified as a dichotomous variable: season 1 /season 2 ) was assessed with Fisher's exact test.
RSVH risk ratios and corresponding 95\% confidence intervals (CIs) were calculated to compare between-group cumulative incidences, and odds ratios (ORs) were computed. All tests were two-tailed, and a $p<0.05$ was assumed to indicate statistical significance. All analyses were run using the SPSS software, version 13.0 (SPSS Inc, Chicago, IL).

\section{Results}

-Fig. 1 shows the patient flow between 2015 and 2017. A total of 632 eligible infants identified from the birth registries at the three centers, 314 in season 1 and 318 in season 2, were considered for this study. Data were unavailable for 96 patients ( 52 in season 1 and 44 in season 2) because parents could not be interviewed or contacted, or refused consent. The sample included in the final analysis thus consisted of 536 infants (262 in season 1 and 274 in season 2).

- Table 1 shows the baseline characteristics and demographic features of the infants in the two seasons. There were no significant differences between the two groups in terms of mean gestational age, birth weight, multiple pregnancies, need for mechanical ventilation during NICU stay, proportion of infants with HSCHD, or risk factor scores. The only difference concerned the proportion of infants discharged with a diagnosis of BPD, which was significantly higher in the second season.

The proportion of infants was not exposed to palivizumab increased significantly from $63.7 \%$ in season 1 to $80.6 \%$ in season 2 ( $p$-value $<0.0001$ ). Similarly the proportion of infants hospitalized for confirmed RSV-related respiratory infections rose from 5 (1.9\%) of 262 infants in season 1 to 14 (5.1\%) of 274 infants in season $2(\mathrm{OR}=2.77 ; 95 \% \mathrm{Cl}$ : 0.98-7.78; $p$-value $=0.045)$. When only the infants not exposed to palivizumab were considered, a similar increase emerged from 3 (1.8\%) of 167 infants in season 1 to $13(5.9 \%)$ of 221 infants in season 2 $(\mathrm{OR}=3.42 ; 95 \% \mathrm{CI}$ : 0.96-12.20; $p$-value $=0.044)$.

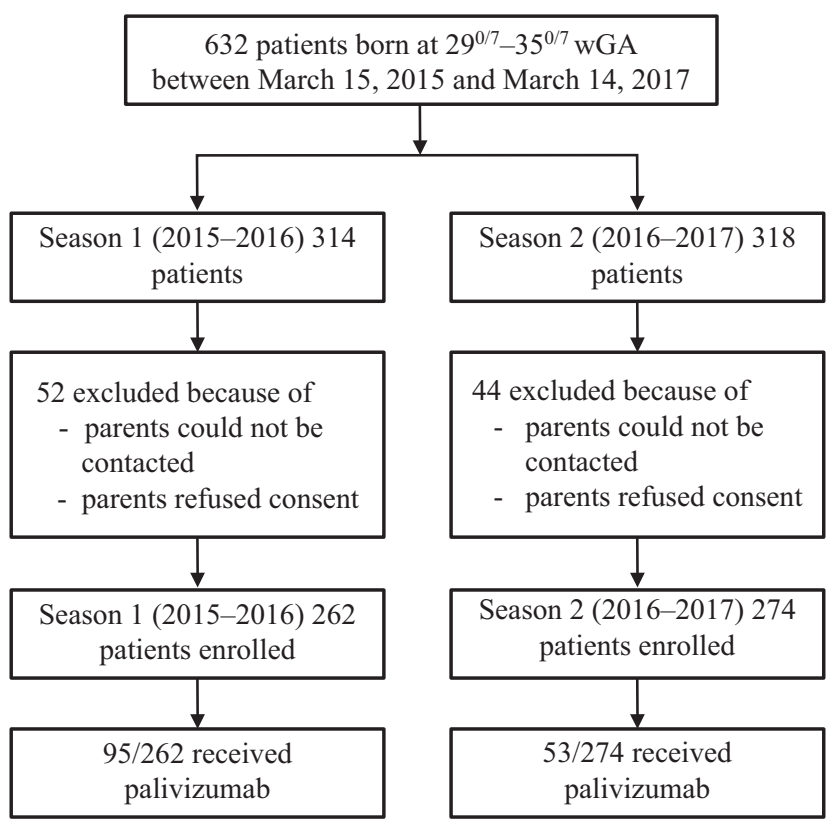

Fig. 1 Study flow chart. wGA, weeks of gestational age. 
Table 1 Infant baseline characteristics and hospital admissions for respiratory infections proportions

\begin{tabular}{|c|c|c|c|c|c|}
\hline & $\begin{array}{l}\text { Season } 1 \\
2015-2016 \\
n=262(\%)\end{array}$ & $\begin{array}{l}\text { Season } 2 \\
2016-2017 \\
n=274(\%)\end{array}$ & OR & $95 \% \mathrm{Cl}$ & $p$-Value \\
\hline Gestational age (days), mean $( \pm S D)$ & $229( \pm 10.9)$ & $228( \pm 11.6)$ & - & - & 0.54 \\
\hline Birth weight (grams), mean (SD) & $1,803( \pm 441.4)$ & $1,768( \pm 492.9)$ & - & - & 0.39 \\
\hline $\begin{array}{l}\text { Mechanical ventilation during } \\
\text { NICU stay (at least } 24 \text { hours) }\end{array}$ & $101 / 262(38.5)$ & $106 / 274(38.6)$ & - & - & 0.98 \\
\hline CLD & $0 / 262(0)$ & $12 / 274(4.4)$ & - & - & $<0.001$ \\
\hline HSCHD & $6 / 262(2.3)$ & $8 / 274(2.92)$ & - & - & 0.37 \\
\hline Multiple pregnancies & $87 / 262(33.2)$ & $92 / 274(33.6)$ & - & - & 0.92 \\
\hline Patients not exposed to palivizumab & $167 / 262(63.7)$ & $221 / 274(80.6)$ & - & - & $<0.001$ \\
\hline Risk factor score ${ }^{a}$ & & & - & - & 0.85 \\
\hline 0 & $28 / 262(10.7)$ & $29 / 274(10.6)$ & - & - & - \\
\hline 1 & $83 / 262(31.7)$ & $95 / 274(34.7)$ & - & - & - \\
\hline 2 & $91 / 262(34.7)$ & $95 / 274(34.7)$ & - & - & - \\
\hline 3 & $51 / 262(19.5)$ & $44 / 274(16.1)$ & - & - & - \\
\hline 4 & $9 / 262(3.4)$ & $11 / 274(4.0)$ & - & - & - \\
\hline RSVH among all infants & $5 / 262(1.91)$ & $14 / 274(5.11)$ & 2.77 & $0,98-7.80$ & 0.045 \\
\hline RSVH among infants not exposed to palivizumab & $3 / 167(1.80)$ & $13 / 221(5.88)$ & 3.42 & $0.96-12.20$ & 0.045 \\
\hline RSVH among infants exposed to palivizumab & $2 / 95(2.10)$ & $1 / 53(1.88)$ & 1.09 & $0.67-1.35$ & 0.88 \\
\hline Hospitalizations for bronchiolitis unrelated to RSV & $11 / 262(4.20)$ & $14 / 274(5.11)$ & - & - & 0.63 \\
\hline
\end{tabular}

Abbreviations: $\mathrm{Cl}$, confidence interval; CLD, chronic lung disease; HSCHD, haemodynamically significant congenital heart disease; NICU, neonatal intensive care unit; OR, odds ratio; RSV, respiratory syncytial virus; RSVH, hospitalization due to RSV; SD, standard deviation.

Note: Data are unless otherwise stated as $\mathrm{n}(\%)$.

${ }^{a}$ Risk factor score was calculated, scoring one point for each of the following: (1) more than two siblings at home; (2) daycare attendance; (3) no breastfeeding, or breastfeeding for less than 2 months; (4) parental smoking; and (5) discharge from the NICU in the epidemic season.

In contrast, there was no significant difference between the two seasons in the incidence of hospital admissions for low respiratory tract infection caused by respiratory viruses other than RSV.

In accordance with AIFA and scientific society recommendations, all infants with BPD in either season were given palivizumab. Only one episode of RSVH occurred among these infants, and it happened in season 1 . This clearly means that there was no impact on the above-mentioned results attributable to the increase in the number of BPD patients in season 2 , as previously reported.

It is worth noting that the 14 episodes of RSVH occurring in season 2 affected infants with gestational ages of 31 weeks (three cases), 32 weeks (two cases), 33 weeks (two cases), 34 weeks (two cases), and 35 weeks (five cases), whereas the five episodes occurring in season 1 involved infants with gestational ages of 31 weeks (one case), 33 weeks (three cases), and 34 weeks (one case).

\section{Discussion}

The present study assessed the burden of RSVH for infants born at 29 to $35 \mathrm{wGA}$ and discharged from three NICUs in northern Italy during two consecutive seasons in which two different palivizumab prophylaxis allocation policies were adopted following changes to AIFA's reimbursement policies. It emerged that the overall incidence of RSVH increased significantly in the season 2 , when eligibility for prophylaxis with palivizumab free of charge was restricted to infants born $<29$ wGA and denied to those with gestational ages between 29 and 35 weeks.

Our results confirm and reinforce similar reports from other countries and health care settings. In the U.S.A., Rajah et al found a significant increase in the number of RSVH involving infants up to 6 months old born at 29 to $34^{6 / 7}$ wGA after the implementation of the 2014 recommendations of the American Academy of Pediatrics, as well as an increased morbidity (in terms of the need for oxygen supplementation, admission to pediatric intensive care units, mechanical ventilation, and length of hospital stay) in 29 to $34^{6 / 7}$ wGA in $<3$ months old infants. ${ }^{12}$ Likewise, in a single-center study from Italy that retrospectively reviewed the records for children $\leq 12$ months old admitted to hospital for RSV-induced lower respiratory tract infections from 2014 to 2017, there was a higher proportion of admissions for infants born $<36 \mathrm{wGA}$, and a greater need for high-flow nasal cannula ventilation in the 2016 to 2017 epidemic season (after more restrictive palivizumab allocation policies had been implemented) than in the previous two seasons. ${ }^{13}$ In Italy, the birth rate is approximately $450,000 / y e a r$, and it is estimated that some $5 \%$ of infants are 
born at between 29 and $35 \mathrm{wGA}$, that is, approximately 22,500 newborns. Assuming a 3.2\% increase in RSVH, as suggested by the results of our analysis, we should expect an increase of approximately 720 admissions in a year in this subgroup of premature infants. Though a thorough economic analysis goes beyond the scope of this study, our findings indicate that it is mandatory to consider the health care costs resulting from excessively restrictive rules covering the prescription of palivizumab. Our conclusion is consistent with the report from Zuccotti and Fabiano, who performed an economic impact analysis on RSV-related hospitalization and clinical examinations for infants $<3$ years old, and concluded that extending the prophylaxis to infants born at 29 to 32 wGA up until they are 6 months old is a cost-effective strategy. ${ }^{6}$ Besides there is the indirect burden of RSV-related infections to consider, including social costs, parental burden, ${ }^{14}$ and long-term respiratory sequelae. It is well known that RSV infection, especially when severe, is a risk factor for recurrent wheezing, asthma, and overall lung function impairment. The multicenter, randomized controlled MAKI trial performed by the Dutch RSV Neonatal Network showed a 61\% relative reduction in the number of wheezing days during the 1st year of life in infants born at 33 to $35 \mathrm{wGA}$ who were exposed to palivizumab as opposed to placebo. ${ }^{15}$ Mochizuki et al also documented a significant reduction in the prevalence of wheezing at 6 years of age in a group treated with palivizumab versus placebo (15.3 and $31.6 \%){ }^{16}$ These data point to the real advisability of preventing severe RSV-related infections in infants (especially those at high risk) to limit late negative outcomes of early exposure to this pathogen.

It is worth emphasizing that the restrictions on eligibility for palivizumab reimbursement in season 2 led to a significant increase in $\mathrm{RSVH}$, but had no impact on hospital admissions for other respiratory viruses. This is important in that it rules out the possible influence, for instance, of other environmental conditions relating to respiratory viruses circulating in the community, or of an overall higher risk of respiratory disorders in the study population in season 2 , to justify the excess number of RSVH.

\section{Limitations}

We acknowledge that our study has some limitations. Due to the small size of the samples of infants, who experienced an episode of RSVH, it was impossible to stratify the risk by gestational age at birth. In addition, data collection was based on a search for birth data and telephone interviews, so the study is prone to recall bias. We are, nonetheless, convinced that a hospital admission is a memorable event for an infant's parents and unlikely to be overlooked by the questionnaire that we administered to parents, whose compliance in answering the interview questions and coming to the center in the event of hospitalization was generally good.

\section{Conclusion}

In conclusion, the results of our study confirm previouslypublished evidence, and underscore the fact that all pretermborn infants, regardless of their gestational age, are highly vulnerable to severe RSV-related infections. In addition, this study shows that the incidence of RSVH increases significantly when eligibility for palivizumab prophylaxis is restricted. Clinicians and policy-makers should carefully consider the cost-benefit balance concerning palivizumab prophylaxis, and be aware that a limited coverage is likely to bring a surge in RSVH for infants not receiving it.

\section{Conflict of Interest}

P.M. received speaker fees from Abbvie, Janssen; advisory board fees from Abbvie, Sanofi-Pasteur, Janssen, Medimmune; he is founding member of Respiratory Syncytial Virus Network (ReSViNET) Foundation. E.B. received speaker fees from Abbvie, GSK, Janssen; he is founding member of Respiratory Syncytial Virus Network (ReSViNET) Foundation. All the other authors have nothing to disclose related to this article.

\section{References}

1 Mazur NI, Martinón-Torres F, Baraldi E, et al; Respiratory Syncytial Virus Network (ReSViNET). Lower respiratory tract infection caused by respiratory syncytial virus: current management and new therapeutics. Lancet Respir Med 2015;3(11):888-900

2 Simões EAF, Bont L, Manzoni P, et al. Past, present and future approaches to the prevention and treatment of respiratory syncytial virus infection in children. Infect Dis Ther 2018;7(01): 87-120

3 Andabaka T, Nickerson JW, Rojas-Reyes MX, Rueda JD, Bacic Vrca $\mathrm{V}$, Barsic B. Monoclonal antibody for reducing the risk of respiratory syncytial virus infection in children. Cochrane Database Syst Rev 2013;30(04):CD006602

4 American Academy of Pediatrics Committee on Infectious Diseases; American Academy of Pediatrics Bronchiolitis Guidelines Committee. Updated guidance for palivizumab prophylaxis among infants and young children at increased risk of hospitalization for respiratory syncytial virus infection. Pediatrics 2014; 134(02):e620-e638

5 Bollani L, Baraldi E, Chirico G, et al; Italian Society of Neonatology. Revised recommendations concerning palivizumab prophylaxis for respiratory syncytial virus (RSV). Ital J Pediatr 2015;41:97

6 Zuccotti G, Fabiano V. Indications to respiratory syncytial virus immunoprophylaxis in the 29-32 wGA group: is there still room for debating? Ital J Pediatr 2017;43(01):17

7 Lanari M, Prinelli F, Adorni F, et al; Study Group of Italian Society of Neonatology on Risk Factors for RSV Hospitalization. Risk factors for bronchiolitis hospitalization during the first year of life in a multicenter Italian birth cohort. Ital J Pediatr 2015;41:40

8 Anderson EJ, Krilov LR, DeVincenzo JP, et al. SENTINEL1: An Observational Study of Respiratory Syncytial Virus Hospitalizations among U.S. Infants Born at 29 to 35 Weeks' Gestational Age Not Receiving Immunoprophylaxis. Am J Perinatol 2017;34(01): 51-61

9 Walsh MC, Szefler S, Davis J, et al. Summary proceedings from the bronchopulmonary dysplasia group. Pediatrics 2006;117(3, Pt. 2):S52-S56

10 Feltes TF, Sondheimer HM, Tulloh RM, et al; Motavizumab Cardiac Study Group. A randomized controlled trial of motavizumab versus palivizumab for the prophylaxis of serious respiratory syncytial virus disease in children with hemodynamically significant congenital heart disease. Pediatr Res 2011;70(02): 186-191

11 Yi H, Lanctôt KL, Bont L, et al; CARESS investigators. Respiratory syncytial virus prophylaxis in Down syndrome: a prospective cohort study. Pediatrics 2014;133(06):1031-1037 
12 Rajah B, Sánchez PJ, Garcia-Maurino C, Leber A, Ramilo O, Mejias A. Impact of the updated guidance for palivizumab prophylaxis against respiratory syncytial virus infection: a single center experience. J Pediatr 2017;181:183-188

13 Capizzi A, Silvestri M, Orsi A, Cutrera R, Rossi GA, Sacco O. The impact of the recent AAP changes in palivizumab authorization on RSV-induced bronchiolitis severity and incidence. Ital J Pediatr 2017;43(01):71

14 Carbonell-Estrany X, Dall'Agnola A, Fullarton JR, et al. Interaction between healthcare professionals and parents is a key determinant of parental distress during childhood hospitalisation for respiratory syncytial virus infection (European RSV Outcomes Study [EROS]). Acta Paediatr 2018;107(05):854-860

15 Blanken MO, Rovers MM, Molenaar JM, et al; Dutch RSV Neonatal Network. Respiratory syncytial virus and recurrent wheeze in healthy preterm infants. N Engl J Med 2013;368(19): 1791-1799

16 Mochizuki H, Kusuda S, Okada K, Yoshihara S, Furuya H, Simões EAF; Scientific Committee for Elucidation of Infantile Asthma. Palivizumab prophylaxis in preterm infants and subsequent recurrent wheezing: six year follow-up study. Am J Respir Crit Care Med 2017;196(01):29-38 